\title{
Climate Change, Human Health, and Integrative Research: A Transformative Imperative
}

The past two years mark an historical turning point in the public policy debate on human-driven climate change. Arguments that the evidence is either inconclusive or that bold action is economically unjustified are now clearly in retreat, faced with an increasingly mature science of climate change and mounting evidence of adverse impacts.

Even so, our societies have not yet gotten the full measure of the risks posed by climate change-particularly the risks to health. Nor is it well understood that many risks will be compounded by the actions of coexistent stressors, such as land degradation, water shortages, disruptions to the global cycles of nitrogen and phosphorus, accelerating biodiversity losses, and ocean acidification. Indeed, population health researchers have been slow to engage in this (admittedly complex) arena-and the formal health sector tends, as ever, to see these problems as belonging to the "environment sector."

Well, first, we must stop thinking in outmoded differentiated sectoral terms. Many of today's influences on population health result from the unprecedented pressures that urbanization, long-distance trade, intensified food production, energy generation, landscape transformations, and water engineering are placing on the natural environment. These environmental changes are regional or global in scale; they involve changes in diverse and complex natural systems; their impacts on health are both direct and indirect.

Second, we researchers must respond to this great challenge to elucidate the linkages between environmental changes and human health, and to deploy that information to help motivate policies that mitigate climatic and other environmental changes. Such research requires a broadened ecohealth perspective able to address the diverse interac- tions and feedback processes that mediate the impacts on human health.

This research domain is shown schematically in Figure 1. It illustrates, for climate change, the direct impact pathways and several of the main indirect pathways that affect the risks of infectious diseases and undernutrition. The various categories of environmental changes shown at the left-land use, freshwater, agriculture, oceans, and urbanization-have all changed at historically unprecedented rates in recent decades. Various illustrative pathways indicate how they interact with one another, and how they contribute to the health risks resulting from climate change.

Not shown in Figure 1 is the preexisting inequality of material living conditions and health status in populations around the world. Nor does Figure 1 capture the more acute extremes of environmental conditions that will arise, the local and regional crises and catastrophes that are likely to occur, and the inevitable tensions, conflicts, displacement, misery, depression, and assorted health risks that will result.

Climate change will act mostly as a multiplier of existing health problems. If it doubles child diarrheal death rates, which are high rates to start with, then the absolute impact will be very great. Likewise, existing undernutrition will also be significantly amplified. The Chair of the Intergovernmental Panel on Climate Change (IPCC) has recently been quoted thus: "Agricultural yields will decline, with some countries in Africa losing up to half of their farm output by 2020 . Food security will get worse, and malnutrition and hunger will grow" (Pachauri 2009).

The advent of these environmental changes supports further reasons for urgently reducing poverty and its associated health inequities. It reinforces the need for 


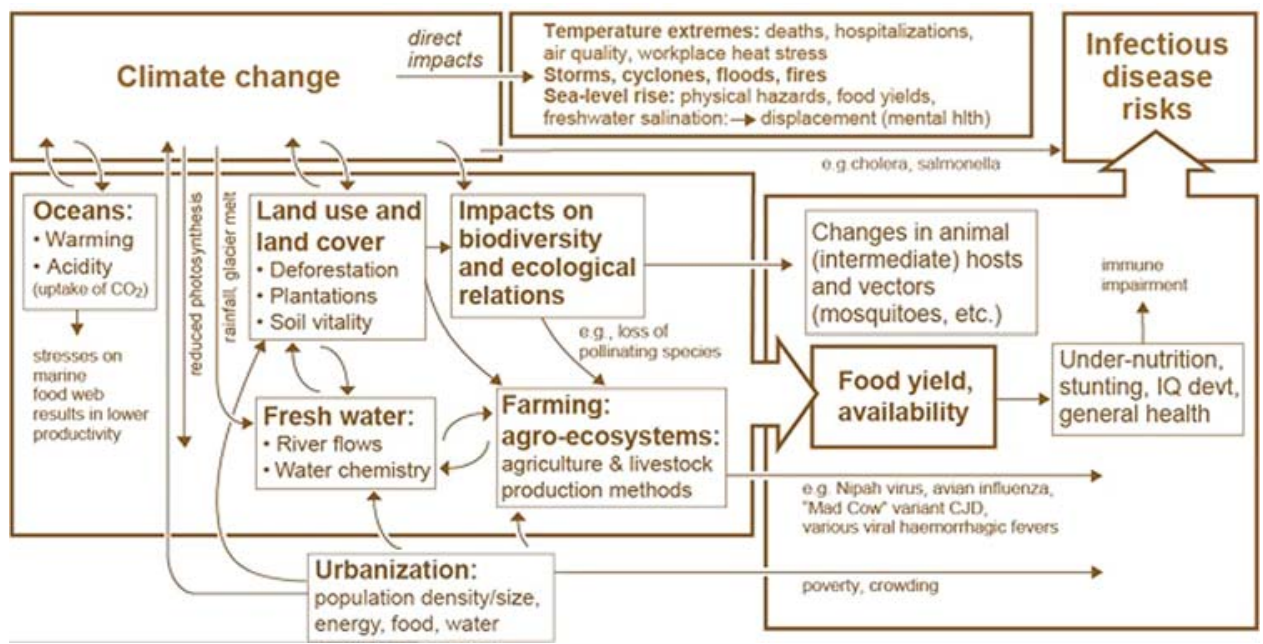

Figure 1. The figure illustrates some of the direct and indirect pathways by which climate change can affect human health. Extreme weather events and sea level rise directly affect health, shown in the top middle compartment. These represent mainly physical stressors that typically produce physical injury and displacement-induced mental health problems. The indirect impacts of climate change on health involve two major categories of different mediating factors (shown by the two lower large boxes). The compartments on the left, such as Oceans, Land Use and Land Cover, etc., represent ecosystem and ecosystem-level processes that interact synergistically via various feedback loops. These influence factors including Food Availability,

economic and social development, and for increased transfer of historically accrued wealth from rich countries to poor countries. And it underscores the need for development strategies to be reframed within the sustainability paradigm. Subsidizing coal-fired power plants or palm-oil plantations in low-income countries is the antithesis of an environmentally sustainable strategy that conserves Earth's life support systems and sustains future population health.

The silver lining to this great cloud that now overhangs population health is that climate change will force our hand, as researchers. We have long intoned ideas about health as a holistic concept, an expression of the full complement of social and ecological factors that constitute human ecology, and about the need to address the "upstream" drivers of health determinants in populations. Now we have the chance, indeed the responsibility, to do that integrated, ecologically attuned, research, and to help illuminate an alternative path to the future. which in turn affects general health. Ecosystem-level processes also affect pathogens, vectors, and reservoirs that drive Infectious Disease Risk. Urbanization is another major factor interacting with climate change and contributing to demographic and socioeconomic conditions that alter disease risk. However, even this relatively complex scheme fails to show all likely interactions and the external drivers, such as greenhouse gas emissions and policies, contributing to regional and local environmental change. Also, while the figure focuses on infectious disease risk as a human health end point, factors like mental health and nutrition also affect chronic disease risk.

Anthony J. McMichael

National Center for Epidemiology and Population Health, The Australian National University, Canberra, ACT, Australia

e-mail: tony.mcmichael@anu.edu.au

Bruce A. Wilcox

Office of Public Health Studies, University of Hawaii at Manoa, Honolulu, HI e-mail: bwilcox@hawaii.edu

\section{REFERENCES}

Pachauri RK (2009) The disappearing deal: American obstacles in Copenhagen. Newsweek, International Edition, October 26, 2009. http://www.newsweek.com/id/218116

Published online: January 16, 2010 